\title{
FREE QUANTITATIVE FOURTH MOMENT THEOREMS ON WIGNER SPACE
}

\author{
SOLESNE BOURGUIN AND SIMON CAMPESE
}

\begin{abstract}
We prove a quantitative Fourth Moment Theorem for Wigner integrals of any order with symmetric kernels, generalizing an earlier result from Kemp et al. (2012). The proof relies on free stochastic analysis and uses a new biproduct formula for bi-integrals. A consequence of our main result is a Nualart-Ortiz-Latorre type characterization of convergence in law to the semicircular distribution for Wigner integrals. As an application, we provide Berry-Esseen type bounds in the context of the free Breuer-Major theorem for the free fractional Brownian motion.
\end{abstract}

\section{INTRODUCTION}

Let $(\mathscr{A}, \varphi)$ be a tracial $W^{*}$-probability space, $S$ be a semicircular random variable and $F=I_{n}(f)$ be a self-adjoint Wigner integral (for a simple example, take off-diagonal homogeneous sums of a semicircular system). Recently, Kemp et al. showed in [KNPS12] that for a sequence of such Wigner integrals, convergence of the fourth moment controls convergence in distribution towards the semicircular law. Moreover, they provided a quantitative bound in terms of the free gradient operator, which is of the form (all unexplained notation appearing in this section will be introduced in the sequel)

$$
d_{\mathcal{C}_{2}}(F, S) \leq \frac{1}{2} \varphi \otimes \varphi\left(\left|\int \nabla_{s}\left(N_{0}^{-1} F\right) \sharp\left(\nabla_{s} F\right)^{*} d s-1 \otimes 1\right|\right) .
$$

Here, $d_{\mathcal{C}_{2}}$ is a distance that metrizes free convergence in distribution (see Definition 2.4), $\nabla$ denotes the free gradient operator first introduced by Biane and Speicher in [BS98] and $N_{0}^{-1}$ stands for the pseudo-inverse of the number operator (see Section 2). In the special case of Wigner integrals of order two, Kemp et al. showed in [KNPS12] that the gradient expression appearing in (1) can further be bounded by the fourth moment. To be more precise, it holds that

$$
\begin{aligned}
d_{\mathcal{C}_{2}}\left(I_{2}(f), S\right) & \leq \frac{1}{2} \varphi \otimes \varphi\left(\left|\int \nabla_{s}\left(N_{0}^{-1} I_{2}(f)\right) \sharp\left(\nabla_{s} I_{2}(f)\right)^{*} d s-1 \otimes 1\right|\right) \\
& \leq \frac{1}{2} \sqrt{\frac{3}{2}} \sqrt{\varphi\left(I_{2}(f)^{4}\right)-2} .
\end{aligned}
$$

2010 Mathematics Subject Classification. 46L54, 68H07, 60H30.

Key words and phrases. Free probability, Wigner integrals, free Malliavin calculus, free stochastic analysis, free quantitative central limit theorems, free Fourth Moment Theorems. 
A question left open in the aforementioned article is whether a similar fourth moment bound holds for Wigner integrals of higher orders, as is the case in the commutative setting (see Nualart and Peccati [NP05] and Nourdin and Peccati[NP09b]). In this paper, we provide a positive answer to this question by proving fourth moment bounds for Wigner integrals of any order with symmetric kernels. Our main result can be paraphrased as follows (see Theorem 3.7 for a precise statement).

Theorem. For a Wigner integral $F$ of order $n$ with normalized symmetric kernel it holds that

$$
\varphi \otimes \varphi\left(\left|\int_{\mathbb{R}_{+}} \nabla_{s}\left(N_{0}^{-1} F\right) \sharp\left(\nabla_{s} F\right)^{*} d s-1 \otimes 1\right|^{2}\right) \leq C_{n}\left(\varphi\left(F^{4}\right)-2\right) .
$$

The constant $C_{n}$ grows asymptotically linearly with $n$ and is a local maximum of a certain polynomial (see Theorem 3.7 for full details). Combined with (1), our result quantifies the free Fourth Moment Theorem [KNPS12, Theorem 1.3] for the case of Wigner integrals with symmetric kernels. In particular, $\sqrt{C_{2}}=\frac{1}{2} \sqrt{\frac{3}{2}}$, so that, by Cauchy-Schwarz, the bound (2) is included as a special case.

It is well-known that in order to ensure that Wigner integrals are self-adjoint (and thus free random variables), the symmetry of the kernel can be relaxed to mirrorsymmetry (see Definition 2.7). As our main bound is stated for symmetric kernels, the natural question arises whether or not it can be generalized to cover the mirrorsymmetric case as well. The answer to this question is negative, as is shown by the counterexample in Remark 3.9.

In the proof of our main result we use a new biproduct formula (see Theorem 3.5) for Wigner bi-integrals (see Subsection 2.2) which generalizes the product formula proved by Biane and Speicher in [BS98] for usual Wigner integrals. In this biproduct formula, the nested contractions become what we call bicontractions. As product formulae play a central role in free (and also classical) stochastic analysis, this might be of independent interest. Other ingredients include the free Malliavin calculus introduced by Biane and Speicher in [BS98] as well as a fine combinatorial analysis. A direct consequence of our bound is a Nualart-Ortiz-Latorre type equivalent condition for convergence towards the semicircular law, which reads as follows (see Theorem 3.10 for a precise statement).

Theorem. A sequence $F_{k}$ of Wigner integrals of order $n$ with normalized symmetric kernels converges in law to the standard semicircular distribution if, and only if,

$$
\int_{\mathbb{R}_{+}}\left(\nabla_{s} F_{k}\right) \sharp\left(\nabla_{s} F_{k}\right)^{*} d s \rightarrow n \cdot 1 \otimes 1 \quad \text { in } \quad L^{2}(\mathscr{A} \otimes \mathscr{A}, \varphi \otimes \varphi) .
$$

This is a free analogue of the main result of [NOL08].

Our findings contribute to the growing literature on free limit theorems obtained by means of free Malliavin calculus and free stochastic analysis. Earlier results include the already mentioned free Fourth Moment Theorem for multiple Wigner integrals [KNPS12], its multidimensional extension [NPS13], the free Fourth Moment Theorem for free Poisson multiple integrals proved in [BP14b] and [Bou16], 
free non-central limit theorems for Wigner and free Poisson integrals obtained in [DN12], [NP13] and [Bou15], as well as limit theorems for the $q$-Brownian motion [DNN13] and convergence of free processes [NT14]. However, all these results, with the exception of [KNPS12] for the case of second order Wigner integrals, are not quantitative. In the commutative setting, which inspired this line of research in the context of free probability theory, the picture is much more complete. Here, quantitative limit theorems exist in the framework of Wiener integrals ([NP05, PT05, NOL08, NP09b, NPR10, NP09a] and references therein), Poisson integrals ([PSTU10, PZ10, Pec11, BP14a, PT13] and references therein) and eigenfunctions of diffusive Markov generators ([Led12, ACP14, CNPP16]).

The rest of this paper is organized as follows: Section 2 introduces the basic concepts of free probability theory and free stochastic analysis. The biproduct formula, our fourth moment bound, as well as the Nualart-Ortiz-Latorre characterization are presented and proved in Section 3. We conclude by providing a Berry-Esseen bound for the free Breuer-Major theorem for the free fractional Brownian motion in Section 4.

\section{Preliminaries}

2.1. Elements of free probability. In the following, a short introduction to free probability theory is provided. For a thorough and complete treatment, see [NS06], [VDN92] and [HP00]. Let $(\mathscr{A}, \varphi)$ be a tracial $W^{*}$-probability space, that is $\mathscr{A}$ is a von Neumann algebra with involution $*$ and $\varphi: \mathscr{A} \rightarrow \mathbb{C}$ is a unital linear functional assumed to be weakly continuous, positive (meaning that $\varphi(X) \geq 0$ whenever $X$ is a non-negative element of $\mathscr{A}$ ), faithful (meaning that $\varphi\left(X X^{*}\right)=0 \Rightarrow X=0$ for every $X \in \mathscr{A}$ ) and tracial (meaning that $\varphi(X Y)=\varphi(Y X)$ for all $X, Y \in \mathscr{A}$ ). The self-adjoint elements of $\mathscr{A}$ will be referred to as random variables. Given a random variable $X \in \mathscr{A}$, the law of $X$ is defined to be the unique Borel measure on $\mathbb{R}$ having the same moments as $X$ (see [NS06, Proposition 3.13]). The noncommutative space $L^{2}(\mathscr{A}, \varphi)$ denotes the completion of $\mathscr{A}$ with respect to the norm $\|X\|_{2}=\sqrt{\varphi\left(X X^{*}\right)}$.

Definition 2.1. A collection of random variables $X_{1}, \ldots, X_{n}$ on $(\mathscr{A}, \varphi)$ is said to be free if

$$
\varphi\left(\left[P_{1}\left(X_{i_{1}}\right)-\varphi\left(P_{1}\left(X_{i_{1}}\right)\right)\right] \cdots\left[P_{m}\left(X_{i_{m}}\right)-\varphi\left(P_{m}\left(X_{i_{m}}\right)\right)\right]\right)=0
$$

whenever $P_{1}, \ldots, P_{m}$ are polynomials and $i_{1}, \ldots, i_{m} \in\{1, \ldots, n\}$ are indices with no two adjacent $i_{j}$ equal.

Let $X \in \mathscr{A}$. The $k$-th moment of $X$ is given by the quantity $\varphi\left(X^{k}\right), k \in \mathbb{N}_{0}$. Now assume that $X$ is a self-adjoint bounded element of $\mathscr{A}$ (in other words, $X$ is a bounded random variable), and write $\rho(X)=\|X\| \in[0, \infty)$ to indicate the spectral radius of $X$.

Definition 2.2. The law (or spectral measure) of $X$ is defined as the unique Borel probability measure $\mu_{X}$ on the real line such that $\int_{\mathbb{R}} P(t) d \mu_{X}(t)=\varphi(P(X))$ for every polynomial $P \in \mathbb{R}[X]$. A consequence of this definition is that $\mu_{X}$ has support in $[-\rho(X), \rho(X)]$. 
The existence and uniqueness of $\mu_{X}$ in such a general framework are proved e.g. in [Tao12, Theorem 2.5.8] (see also [NS06, Proposition 3.13]). Note that, since $\mu_{X}$ has compact support, the measure $\mu_{X}$ is completely determined by the sequence $\left\{\varphi\left(X^{k}\right): k \geq 1\right\}$.

Let $\left\{X_{n}: n \geq 1\right\}$ be a sequence of non-commutative random variables, each possibly belonging to a different non-commutative probability space $\left(\mathscr{A}_{n}, \varphi_{n}\right)$.

Definition 2.3. The sequence $\left\{X_{n}: n \geq 1\right\}$ is said to converge in distribution to a limiting non-commutative random variable $X_{\infty}$ (defined on $\left(\mathscr{A}_{\infty}, \varphi_{\infty}\right)$ ), if $\lim _{n \rightarrow+\infty} \varphi_{n}\left(P\left(X_{n}\right)\right)=\varphi_{\infty}\left(P\left(X_{\infty}\right)\right)$ for every polynomial $P \in \mathbb{R}[X]$.

If $X_{n}, X_{\infty}$ are bounded (and therefore the spectral measures $\mu_{X_{n}}, \mu_{X_{\infty}}$ are welldefined), this last relation is equivalent to saying that

$$
\int_{\mathbb{R}} P(t) \mu_{X_{n}}(d t) \rightarrow \int_{\mathbb{R}} P(t) \mu_{X_{\infty}}(d t) .
$$

An application of the method of moments yields immediately that, in this case, one has also that $\mu_{X_{n}}$ weakly converges to $\mu_{X_{\infty}}$, that is $\mu_{X_{n}}(f) \rightarrow \mu_{X_{\infty}}(f)$, for every $f: \mathbb{R} \rightarrow \mathbb{R}$ bounded and continuous (note that no additional uniform boundedness assumption is needed).

Let $h(x)=\int_{\mathbb{R}} e^{i x \xi} \nu(d \xi)$ be the Fourier transform of a complex measure $\nu$ on $\mathbb{R}$. Note that, as $\nu$ is finite, $h$ is continuous and bounded. For such functions $h$, define the seminorm $\mathscr{I}_{2}(h)$ by

$$
\mathscr{I}_{2}(h)=\int_{\mathbb{R}} \xi^{2}|\nu|(d \xi)
$$

Let $\mathcal{C}_{2}$ denote the set of those functions $h$ for which $\mathscr{I}_{2}(h)<\infty$. Using the seminorm $\mathscr{I}_{2}$ and the set of functions $\mathcal{C}_{2}$, one can define a distance between two self-adjoint random variables.

Definition 2.4. For two self-adjoint random variables $X, Y$, the distance $d_{\mathcal{C}_{2}}(X, Y)$ between $X$ and $Y$ is defined as

$$
d_{\mathcal{C}_{2}}(X, Y)=\sup \left\{|\varphi(h(X))-\varphi(h(Y))|: h \in \mathcal{C}_{2}, \mathscr{I}_{2}(h) \leq 1\right\} .
$$

As is proved in [KNPS12], the distance $d_{\mathcal{C}_{2}}$ is weaker than the Wasserstein distance but still metrizes convergence in law.

Definition 2.5. The centered semicircular distribution with variance $t>0$, denoted by $\mathcal{S}(0, t)$, is the probability distribution given by

$$
\mathcal{S}(0, t)(d x)=(2 \pi t)^{-1} \sqrt{4 t-x^{2}} d x, \quad|x|<2 \sqrt{t} .
$$

Definition 2.6. A free Brownian motion $S$ consists of: (i) a filtration $\left\{\mathscr{A}_{t}: t \geq 0\right\}$ of von Neumann sub-algebras of $\mathscr{A}$ (in particular, $\mathscr{A}_{s} \subset \mathscr{A}_{t}$ for $0 \leq s<t$ ), (ii) a collection $S=\left\{S_{t}: t \geq 0\right\}$ of self-adjoint operators in $\mathscr{A}$ such that: (a) $S_{0}=0$ and $S_{t} \in \mathscr{A}_{t}$ for all $t \geq 0$, (b) for all $t \geq 0, S_{t}$ has a semicircular distribution with mean zero and variance $t$, and (c) for all $0 \leq u<t$, the increment $S_{t}-S_{u}$ is free with respect to $\mathscr{A}_{u}$, and has a semicircular distribution with mean zero and variance $t-u$. 
For every integer $n \geq 1$, the space $L^{2}\left(\mathbb{R}_{+}^{n} ; \mathbb{C}\right)=L^{2}\left(\mathbb{R}_{+}^{n}\right)$ denotes the collection of all complex-valued functions on $\mathbb{R}_{+}^{n}$ that are square-integrable with respect to the Lebesgue measure on $\mathbb{R}_{+}^{n}$.

Definition 2.7. Let $n$ be a natural number and let $f$ be a function in $L^{2}\left(\mathbb{R}_{+}^{n}\right)$.

(1) The adjoint of $f$ is the function $f^{*}\left(t_{1}, \ldots, t_{n}\right)=\overline{f\left(t_{n}, \ldots, t_{1}\right)}$.

(2) The function $f$ is called mirror-symmetric if $f=f^{*}$, i.e., if

$$
f\left(t_{1}, \ldots, t_{n}\right)=\overline{f\left(t_{n}, \ldots, t_{1}\right)}
$$

for almost all $\left(t_{1}, \ldots, t_{n}\right) \in \mathbb{R}_{+}^{n}$ with respect to the product Lebesgue measure.

(3) The function $f$ is called (fully) symmetric if it is real-valued and, for any permutation $\sigma$ in the symmetric group $\mathfrak{S}_{n}$, it holds that $f\left(t_{1}, \ldots, t_{n}\right)=$ $f\left(t_{\sigma(1)}, \ldots, t_{\sigma(n)}\right)$ for almost all $\left(t_{1}, \ldots, t_{n}\right) \in \mathbb{R}_{+}^{n}$ with respect to the product Lebesgue measure.

Definition 2.8. Let $n, m$ be natural numbers and let $f \in L^{2}\left(\mathbb{R}_{+}^{n}\right)$ and $g \in$ $L^{2}\left(\mathbb{R}_{+}^{m}\right)$. Let $p \leq n \wedge m$ be a natural number. The $p$-th nested contraction $f \stackrel{p}{ } g$ of $f$ and $g$ is the $L^{2}\left(\mathbb{R}_{+}^{n+m-2 p}\right)$ function defined by nested integration of the middle $p$ variables in $f \otimes g$ :

$$
\begin{array}{r}
f \stackrel{p}{\sim} g\left(t_{1}, \ldots, t_{n+m-2 p}\right)=\int_{\mathbb{R}_{+}^{p}} f\left(t_{1}, \ldots, t_{n-p}, s_{1}, \ldots, s_{p}\right) \\
g\left(s_{p}, \ldots, s_{1}, t_{n-p+1}, \ldots, t_{n+m-2 p}\right) d s_{1} \cdots d s_{p} .
\end{array}
$$

In the case where $p=0$, the function $f \stackrel{0}{ } g$ is just given by $f \otimes g$.

For $f \in L^{2}\left(\mathbb{R}_{+}^{n}\right)$, we denote by $I_{n}(f)$ the multiple Wigner integral of $f$ with respect to the free Brownian motion as introduced in [BS98]. The space $L^{2}(\mathcal{S}, \varphi)=\left\{I_{n}(f)\right.$ : $\left.f \in L^{2}\left(\mathbb{R}_{+}^{n}\right), n \geq 0\right\}$ is a unital $*$-algebra, with product rule given, for any $n, m \geq 1$, $f \in L^{2}\left(\mathbb{R}_{+}^{n}\right), g \in L^{2}\left(\mathbb{R}_{+}^{m}\right)$, by

$$
I_{n}(f) I_{m}(g)=\sum_{p=0}^{n \wedge m} I_{n+m-2 p}(f \stackrel{p}{\sim} g)
$$

and involution $I_{n}(f)^{*}=I_{n}\left(f^{*}\right)$. For a proof of this formula, see [BS98]. Furthermore, as is well-known, multiple integrals of different orders are orthogonal in $L^{2}(\mathscr{A}, \varphi)$, whereas for two integrals of the same order, the Wigner isometry

$$
\varphi\left(I_{n}(f) I_{n}(g)^{*}\right)=\langle f, g\rangle_{L^{2}\left(\mathbb{R}_{+}^{n}\right)} .
$$

holds.

Remark 2.9. Observe that it follows from the definition of the involution on the algebra $L^{2}(\mathcal{S}, \varphi)$ that operators of the type $I_{n}(f)$ are self-adjoint if and only if $f$ is mirror-symmetric. 
2.2. Bi-integrals and free gradient operator. This subsection introduces the notion of bi-integral and the action of the free gradient operator on Wigner integrals. For a full treatment of these objects, see [BS98].

Let $n, m$ be two positive integers and $f=g \otimes h \in L^{2}\left(\mathbb{R}_{+}^{n}\right) \otimes L^{2}\left(\mathbb{R}_{+}^{m}\right)$. Then, the Wigner bi-integral $I_{n} \otimes I_{m}(f)$ is defined as

$$
I_{n} \otimes I_{m}(f)=I_{n}(g) \otimes I_{m}(h) .
$$

This definition is extended linearly to generic elements $f \in L^{2}\left(\mathbb{R}_{+}^{n}\right) \otimes L^{2}\left(\mathbb{R}_{+}^{m}\right) \cong$ $L^{2}\left(\mathbb{R}_{+}^{n+m}\right)$. From the Wigner isometry (4) for multiple integrals, we obtain the so called Wigner bisometry: for $f \in L^{2}\left(\mathbb{R}_{+}^{n}\right) \otimes L^{2}\left(\mathbb{R}_{+}^{m}\right)$ and $g \in L^{2}\left(\mathbb{R}_{+}^{n^{\prime}}\right) \otimes L^{2}\left(\mathbb{R}_{+}^{m^{\prime}}\right)$ it holds that

$$
\varphi \otimes \varphi\left(I_{n} \otimes I_{m}(f) I_{n^{\prime}} \otimes I_{m^{\prime}}(g)^{*}\right)= \begin{cases}\langle f, g\rangle_{L^{2}\left(\mathbb{R}_{+}^{n}\right) \otimes L^{2}\left(\mathbb{R}_{+}^{m}\right)} & \text { if } n=n^{\prime} \text { and } m=m^{\prime}, \\ 0 & \text { otherwise }\end{cases}
$$

Remark 2.10. Observe that, for any natural numbers $n, m$ and any function $g \otimes h \in$ $L^{2}\left(\mathbb{R}_{+}^{n}\right) \otimes L^{2}\left(\mathbb{R}_{+}^{m}\right)$, it holds that

$$
\begin{aligned}
I_{n} \otimes I_{m}(g \otimes h)^{*} & =\left(I_{n}(g) \otimes I_{m}(h)\right)^{*}=I_{n}(g)^{*} \otimes I_{m}(h)^{*} \\
& =I_{n}\left(g^{*}\right) \otimes I_{m}\left(h^{*}\right)=I_{n} \otimes I_{m}\left((g \otimes h)^{*}\right),
\end{aligned}
$$

so that the operator $I_{n} \otimes I_{m}(g \otimes h)$ is self-adjoint if and only if both the function $g$ and $h$ are mirror-symmetric. By continuous extension (using the Wigner bisometry (5)), it holds that for any fully symmetric function $f \in L^{2}\left(\mathbb{R}_{+}^{n}\right) \otimes L^{2}\left(\mathbb{R}_{+}^{m}\right)$, the operator $I_{n} \otimes I_{m}(f)$ is self-adjoint.

Let $(\mathscr{A}, \varphi)$ be a $W^{*}$-probability space. An $\mathscr{A} \otimes \mathscr{A}$-valued stochastic process $t \mapsto U_{t}$ is called a biprocess. For $p \geq 1, U$ is an element of $\mathscr{B}_{p}$, the space of $L^{p}$-biprocesses, if its norm

is finite.

$$
\|U\|_{\mathscr{B}_{p}}^{2}=\int_{0}^{\infty}\left\|U_{t}\right\|_{L^{p}(\mathscr{A} \otimes \mathscr{A}, \varphi \otimes \varphi)}^{2} d t
$$

The free gradient operator $\nabla: L^{2}(\mathcal{S}, \varphi) \rightarrow \mathscr{B}_{2}$ is a densely-defined and closable operator whose action on Wigner integrals is given by

$$
\nabla_{t} I_{n}(f)=\sum_{k=1}^{n} I_{k-1} \otimes I_{n-k}\left(f_{t}^{(k)}\right)
$$

where $f_{t}^{(k)}\left(x_{1}, \ldots, x_{n-1}\right)=f\left(x_{1}, \ldots, x_{k-1}, t, x_{k}, \ldots, x_{n-1}\right)$ is viewed as an element of $L^{2}\left(\mathbb{R}_{+}^{k-1}\right) \otimes L^{2}\left(\mathbb{R}_{+}^{n-k}\right)$.

Remark 2.11. For general elements of $L^{2}(\mathcal{S}, \varphi)$ in its domain, the free gradient is customarily defined via a Fock space construction (see [BS98]). This level of generality will not be needed in the sequel.

We will also make use of the pseudo-inverse of the number operator $N_{0}^{-1}$, whose action on a multiple Wigner integral of order $n \geq 1$ is given by $N_{0}^{-1} I_{n}(f)=$ $\frac{1}{n} I_{n}(f)$. 
Before concluding this section, we introduce $\sharp$ to be the associative action of $\mathscr{A} \otimes$ $\mathscr{A}^{\mathrm{op}}$ (where $\mathscr{A}^{\mathrm{op}}$ denotes the opposite algebra) on $\mathscr{A} \otimes \mathscr{A}$, as

$$
(A \otimes B) \sharp(C \otimes D)=(A C) \otimes(D B) .
$$

Furthermore, we also write $\sharp$ to denote the action of $\mathscr{A} \otimes L^{2}\left(\mathbb{R}_{+}\right) \otimes \mathscr{A}^{\text {op }}$ on $\mathscr{A} \otimes$ $L^{2}\left(\mathbb{R}_{+}\right) \otimes \mathscr{A}$, as

$$
(A \otimes f \otimes B) \sharp(C \otimes g \otimes D)=(A C) \otimes f g \otimes(D B) .
$$

The multiplication $\sharp$ naturally appears in the following bound from [KNPS12] on the $d_{\mathcal{C}_{2}}$ distance introduced above.

Theorem 2.12 ([KNPS12]). Let $S$ be a standard semicircular random variable and $F \in L^{2}(\mathcal{S}, \varphi)$ be self-adjoint, in the domain of the free gradient $\nabla$ and such that $\varphi(F)=0$. Then,

$$
d_{\mathcal{C}_{2}}(F, S) \leq \frac{1}{2} \varphi \otimes \varphi\left(\left|\int_{\mathbb{R}_{+}} \nabla_{s}\left(N_{0}^{-1} F\right) \sharp\left(\nabla_{s} F\right)^{*} d s-1 \otimes 1\right|\right) .
$$

\section{MAIN RESUlTS}

3.1. Bicontractions and biproduct formula. As announced in the introduction, we will need an extension of the product formula (3) from [BS98]. To this end, we introduce the notion of bicontraction.

Definition 3.1. Let $n_{1}, m_{1}, n_{2}, m_{2}$ be positive integers. Let $f \in L^{2}\left(\mathbb{R}_{+}^{n_{1}}\right) \otimes$ $L^{2}\left(\mathbb{R}_{+}^{m_{1}}\right) \cong L^{2}\left(\mathbb{R}_{+}^{n_{1}+m_{1}}\right)$ and $g \in L^{2}\left(\mathbb{R}_{+}^{n_{2}}\right) \otimes L^{2}\left(\mathbb{R}_{+}^{m_{2}}\right) \cong L^{2}\left(\mathbb{R}_{+}^{n_{2}+m_{2}}\right)$ and let $p \leq n_{1} \wedge n_{2}, r \leq m_{1} \wedge m_{2}$ be natural numbers. The $(p, r)$-bicontraction $f \stackrel{p, r}{\sim} g$ is the $L^{2}\left(\mathbb{R}_{+}^{n_{1}+n_{2}-2 p}\right) \otimes L^{2}\left(\mathbb{R}_{+}^{m_{1}+m_{2}-2 r}\right) \cong L^{2}\left(\mathbb{R}_{+}^{n_{1}+n_{2}+m_{1}+m_{2}-2 p-2 r}\right)$ function defined by

$$
\begin{aligned}
& f \stackrel{p, r}{\stackrel{2}{r}} g\left(t_{1}, \ldots, t_{n_{1}+n_{2}+m_{1}+m_{2}-2 p-2 r}\right) \\
& =\int_{\mathbb{R}_{+}^{p+r}} f\left(t_{1}, \ldots, t_{n_{1}-p}, s_{p}, \ldots, s_{1}, y_{1}, \ldots, y_{r},\right. \\
& \left.t_{n_{1}+n_{2}+m_{2}-2 p-r+1}, \ldots, t_{n_{1}+n_{2}+m_{1}+m_{2}-2 p-2 r}\right) \\
& \times g\left(s_{1}, \ldots, s_{p}, t_{n_{1}-p+1}, \ldots, t_{n_{1}+n_{2}+m_{2}-2 p-r}, y_{r}, \ldots, y_{1}\right) \\
& d s_{1} \cdots d s_{p} d y_{1} \cdots d y_{r} .
\end{aligned}
$$

Remark 3.2. Observe that for $f=f_{1} \otimes f_{2}$ and $g=g_{1} \otimes g_{2}$ with $f_{1} \in L^{2}\left(\mathbb{R}_{+}^{n_{1}}\right)$, $f_{2} \in L^{2}\left(\mathbb{R}_{+}^{m_{1}}\right), g_{1} \in L^{2}\left(\mathbb{R}_{+}^{n_{2}}\right)$ and $g_{2} \in L^{2}\left(\mathbb{R}_{+}^{m_{2}}\right)$, the above definition reads

$$
f \stackrel{p, r}{r} g=\left(f_{1} \otimes f_{2}\right) \stackrel{p, r}{\sim}\left(g_{1} \otimes g_{2}\right)=\left(f_{1} \stackrel{p}{\sim} g_{1}\right) \otimes\left(g_{2} \stackrel{r}{\sim} f_{2}\right),
$$

where the contractions appearing on the right-hand side are the nested contractions introduced in Definition 2.8.

Remark 3.3. In what follows, for $f, g$ as in Definition 3.1, we write $f \stackrel{p, r}{\stackrel{2}{g}} g$ and $f \stackrel{s}{S} g$ to denote the bicontraction and contraction of $f$ and $g$, respectively. Here, we have somewhat abused notation by using the same symbol for a function living in $L^{2}\left(\mathbb{R}_{+}^{n_{1}}\right) \otimes L^{2}\left(\mathbb{R}_{+}^{m_{1}}\right)$ or its identification in $L^{2}\left(\mathbb{R}_{+}^{n_{1}+m_{1}}\right)$. However, it will always 
be clear from the type of contraction used which version of the function is being considered.

The following result collects some properties of bicontractions in the case where both functions are symmetric.

Lemma 3.4. For $n_{1}, m_{1}, n_{2}, m_{2} \in \mathbb{N}$, let $f \in L^{2}\left(\mathbb{R}_{+}^{n_{1}}\right) \otimes L^{2}\left(\mathbb{R}_{+}^{m_{1}}\right) \cong L^{2}\left(\mathbb{R}_{+}^{n_{1}+m_{1}}\right)$ and $g \in L^{2}\left(\mathbb{R}_{+}^{n_{2}}\right) \otimes L^{2}\left(\mathbb{R}_{+}^{m_{2}}\right) \cong L^{2}\left(\mathbb{R}_{+}^{n_{2}+m_{2}}\right)$ be fully symmetric functions. Furthermore, let $p \leq n_{1} \wedge n_{2}$ and $r \leq m_{1} \wedge m_{2}$ be natural numbers such that $p+r=p^{\prime}+r^{\prime}$. Then, the following is true.

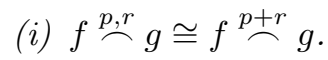

(ii) $f \stackrel{p, r}{\sim} g=f \stackrel{p^{\prime}, r^{\prime}}{\sim} g$.

(iii) $\|f \stackrel{p, r}{\sim} g\|_{L^{2}\left(\mathbb{R}_{+}^{n_{1}+n_{2}-2 p}\right) \otimes L^{2}\left(\mathbb{R}_{+}^{m_{1}+m_{2}-2 r}\right)}^{2}=\|f \stackrel{p+r}{\sim} g\|_{L^{2}\left(\mathbb{R}_{+}^{n_{1}+n_{2}+m_{1}+m_{2}-2 p-2 r}\right)}^{2}$.

(iv) $f \stackrel{n_{1}, m_{1}}{\sim} f=\|f\|_{L^{2}\left(\mathbb{R}_{+}^{n_{1}}\right) \otimes L^{2}\left(\mathbb{R}_{+}^{m_{1}}\right)}^{2} 1 \otimes 1$, which is a constant in $L^{2}\left(\mathbb{R}_{+}^{n_{1}}\right) \otimes$ $L^{2}\left(\mathbb{R}_{+}^{m_{1}}\right)$.

Proof. Just exploit the full symmetry of $f$ in the above definition of contractions.

We are now ready to state the biproduct formula, which will be a crucial tool in order to prove our main result.

Theorem 3.5. For $n_{1}, m_{1}, n_{2}, m_{2} \in \mathbb{N}$, let $f \in L^{2}\left(\mathbb{R}_{+}^{n_{1}}\right) \otimes L^{2}\left(\mathbb{R}_{+}^{m_{1}}\right) \cong L^{2}\left(\mathbb{R}_{+}^{n_{1}+m_{1}}\right)$ and $g \in L^{2}\left(\mathbb{R}_{+}^{n_{2}}\right) \otimes L^{2}\left(\mathbb{R}_{+}^{m_{2}}\right) \cong L^{2}\left(\mathbb{R}_{+}^{n_{2}+m_{2}}\right)$. Then it holds that

(9) $I_{n_{1}} \otimes I_{m_{1}}(f) \sharp I_{n_{2}} \otimes I_{m_{2}}(g)=\sum_{p=0}^{n_{1} \wedge n_{2}} \sum_{r=0}^{m_{1} \wedge m_{2}} I_{n_{1}+n_{2}-2 p} \otimes I_{m_{1}+m_{2}-2 r}(f \stackrel{p, r}{\sim} g)$.

Proof. Using a density argument together with the bisometry property of Wigner bi-integrals, it is enough to prove the claim for functions $f$ and $g$ of the type $a \otimes b$ where $a \in L^{2}\left(\mathbb{R}_{+}^{n}\right)$ and $b \in L^{2}\left(\mathbb{R}_{+}^{m}\right)$ as the subset of functions

$$
\left\{a \otimes b: a \in L^{2}\left(\mathbb{R}_{+}^{n}\right), b \in L^{2}\left(\mathbb{R}_{+}^{m}\right)\right\}
$$

is dense in $\left.L^{2}\left(\mathbb{R}_{+}^{n}\right) \otimes L^{2}\left(\mathbb{R}_{+}^{m}\right)\right)$. Let therefore $f=a \otimes b$ with $a \in L^{2}\left(\mathbb{R}_{+}^{n_{1}}\right)$, $b \in L^{2}\left(\mathbb{R}_{+}^{m_{1}}\right)$ and $g=c \otimes d$ with $c \in L^{2}\left(\mathbb{R}_{+}^{n_{2}}\right), d \in L^{2}\left(\mathbb{R}_{+}^{m_{2}}\right)$. It holds that

$$
\begin{aligned}
I_{n_{1}} \otimes I_{m_{1}}(a \otimes b) \sharp I_{n_{2}} \otimes I_{m_{2}}(c \otimes d) & =I_{n_{1}}(a) \otimes I_{m_{1}}(b) \sharp I_{n_{2}}(c) \otimes I_{m_{2}}(d) \\
& =I_{n_{1}}(a) \cdot I_{n_{2}}(c) \otimes I_{m_{1}}(d) \cdot I_{m_{2}}(b) .
\end{aligned}
$$


Using the usual multiplication formula for Wigner integrals on both sides of the tensor product, we get

$$
\begin{aligned}
& I_{n_{1}}(a) \cdot I_{n_{2}}(c) \otimes I_{m_{1}}(d) \cdot I_{m_{2}}(b) \\
& =\left(\sum_{p=0}^{n_{1} \wedge n_{2}} I_{n_{1}+n_{2}-2 p}(a \stackrel{p}{\sim} c)\right) \otimes\left(\sum_{r=0}^{m_{1} \wedge m_{2}} I_{m_{1}+m_{2}-2 r}(d \stackrel{r}{\sim} b)\right) \\
& =\sum_{p=0}^{n_{1} \wedge n_{2}} \sum_{r=0}^{m_{1} \wedge m_{2}} I_{n_{1}+n_{2}-2 p} \otimes I_{m_{1}+m_{2}-2 r}((a \stackrel{p}{\sim} c) \otimes(d \stackrel{r}{\sim} b))
\end{aligned}
$$

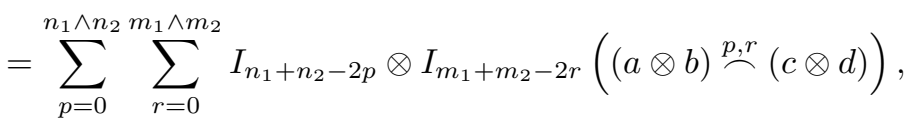

where the last equality follows from the identity (8).

\section{Remark 3.6.}

1. By taking $m_{1}=m_{2}=0, f=u \otimes 1$ and $g=v \otimes 1$, we recover the usual product formula (3) for Wigner integrals.

2. Note that a similar version of the above biproduct formula also holds for the usual tensor product (with a slightly different definition for the bicontractions). Furthermore, using the same methodology, one could also define contractions and product formulae for higher order tensors.

3.2. Quantitative Fourth Moment Theorems. We are now in the position of stating the main result of this paper, namely a bound on the quantity appearing in the right hand side of (7) in terms of the fourth moment, which then leads to a quantitative Fourth Moment Theorem for multiple Wigner integrals.

Theorem 3.7. For $n \in \mathbb{N}$, let $F=I_{n}(f)$ be a Wigner integral of order $n$ with $f \in L^{2}\left(\mathbb{R}_{+}^{n}\right)$ symmetric and such that $\|f\|_{L^{2}\left(\mathbb{R}_{+}^{n}\right)}^{2}=1$. Then, it holds that

$$
\varphi \otimes \varphi\left(\left|\int_{\mathbb{R}_{+}} \nabla_{s}\left(N_{0}^{-1} F\right) \sharp\left(\nabla_{s} F\right)^{*} d s-1 \otimes 1\right|^{2}\right) \leq C_{n}\left(\varphi\left(F^{4}\right)-2\right),
$$

where $C_{n}=\frac{1}{n^{2}} \max \left\{P_{n}\left(\left\lfloor u_{0}\right\rfloor\right), P_{n}\left(\left\lceil u_{0}\right\rceil\right)\right\}$ with

$$
\begin{aligned}
P_{n}(u) & =\frac{1}{3} u^{2}(n-u+1)\left(2(n-u)^{2}+4(n-u)+3\right), \\
u_{0} & =\frac{1}{5}\left(4(n+1)-\frac{r(n)}{\sqrt[3]{4}}-\frac{2 n^{2}+4 n-3}{\sqrt[3]{2} r(n)}\right)
\end{aligned}
$$

and

$$
r(n)=\sqrt[3]{4 n^{3}+12 n^{2}+5 \sqrt{2} \sqrt{4 n^{4}+16 n^{3}+20 n^{2}+8 n+5}+22 n+14} .
$$

Proof. In the following we will use the shorthand $f_{s}^{(k)}$ to denote the function given by

$$
f_{s}^{(k)}\left(x_{1}, \ldots, x_{n-1}\right)=f\left(x_{1}, \ldots, x_{k-1}, s, x_{k+1}, \ldots, x_{n}\right) .
$$


Observe that

$$
\begin{array}{rl}
\int_{\mathbb{R}_{+}}\left(\nabla_{s} F\right) \sharp\left(\nabla_{s} F\right)^{*} & d s \\
& =\sum_{k, q=1}^{n} \int_{\mathbb{R}_{+}} I_{k-1} \otimes I_{n-k}\left(f_{s}^{(k)}\right) \sharp\left(I_{q-1} \otimes I_{n-q}\left(f_{s}^{(q)}\right)\right)^{*} d s \\
& =\sum_{k, q=1}^{n} \int_{\mathbb{R}_{+}} I_{k-1} \otimes I_{n-k}\left(f_{s}^{(k)}\right) \sharp I_{q-1} \otimes I_{n-q}\left(f_{s}^{(q)}\right) d s,
\end{array}
$$

where the last equality follows from the full symmetry of the function $f$. Using the product formula for bi-integrals proven in Theorem 3.5 yields

$$
\begin{aligned}
\int_{\mathbb{R}_{+}}\left(\nabla_{s} F\right) & \sharp\left(\nabla_{s} F\right)^{*} d s \\
& =\sum_{k, q=1}^{n} \int_{\mathbb{R}_{+}} \sum_{p=0}^{(k \wedge q)-1} \sum_{r=0}^{n-(k \vee q)} I_{k+q-2 p-2} \otimes I_{2 n-k-q-2 r}\left(f_{s}^{(k)} \stackrel{p, r}{\sim} f_{s}^{(q)}\right) d s,
\end{aligned}
$$

and by a Fubini argument one gets

$$
\begin{aligned}
\int_{\mathbb{R}_{+}}\left(\nabla_{s} F\right) \sharp\left(\nabla_{s} F\right)^{*} d s \\
\quad=\sum_{k, q=1}^{n} \sum_{p=0}^{(k \wedge q)-1} \sum_{r=0}^{n-(k \vee q)} I_{k+q-2 p-2} \otimes I_{2 n-k-q-2 r}\left(\int_{\mathbb{R}_{+}} f_{s}^{\left.(k) \stackrel{p, r}{~} f_{s}^{(q)} d s\right) .}\right.
\end{aligned}
$$

The full symmetry of $f$ implies that $f_{s}^{(k)}=f_{s}^{(q)}$ for any $1 \leq k, q \leq n$, which together with Lemma 3.4 yields $\int_{\mathbb{R}_{+}} f_{s}^{(k)} \stackrel{p, r}{\sim} f_{s}^{(q)} d s=f \stackrel{p+r+1}{\sim} f$. Hence,

$$
\int_{\mathbb{R}_{+}}\left(\nabla_{s} F\right) \sharp\left(\nabla_{s} F\right)^{*} d s=\sum_{k, q=1}^{n} \sum_{p=0}^{(k \wedge q)-1} \sum_{r=0}^{n-(k \vee q)} I_{k+q-2 p-2} \otimes I_{2 n-k-q-2 r}(f \stackrel{p+r+1}{\sim} f) .
$$

Exactly those summands $I_{k+q-2 p-2} \otimes I_{2 n-k-q-2 r}(f \stackrel{p+r+1}{\sim} f)$ for which $k+q-$ $2 p-2=0$ and $2 n-k-q-2 r=0$ yield the constant term $\|f\|_{L^{2}\left(\mathbb{R}_{+}^{n}\right)}^{2} \cdot 1 \otimes 1$ (i.e. a constant in $L^{2}\left(\mathbb{R}_{+}\right) \otimes L^{2}\left(\mathbb{R}_{+}\right)$). These conditions, along with the ranges of summation, imply that $k=q$ and $p+r+1=n$. Therefore, fixing $k$, for which we have $n$ possibilities, fixes the other three indices $q, p$ and $r$ to take the values $k, k-1$ and $n-k$, respectively. Recalling that $\|f\|_{L^{2}\left(\mathbb{R}_{+}^{n}\right)}^{2}=1$, (12) can thus be rewritten as

$$
\begin{aligned}
\int_{\mathbb{R}_{+}}\left(\nabla_{s} F\right) \sharp\left(\nabla_{s} F\right)^{*} d s & \\
=n \cdot 1 \otimes 1+\sum_{k, q=1}^{n} & \sum_{p=0}^{(k \wedge q)-1} \sum_{r=0}^{n-(k \vee q)} \mathbb{1}_{\{n-1-p-r>0\}} \\
& \times I_{k+q-2 p-2} \otimes I_{2 n-k-q-2 r}(f \stackrel{p+r+1}{\sim} f),
\end{aligned}
$$


which, by using that $N_{0}^{-1} F=\frac{1}{n} F$, gives

$$
\begin{aligned}
& \int_{\mathbb{R}_{+}} \nabla_{s}(\left.N_{0}^{-1} F\right) \sharp\left(\nabla_{s} F\right)^{*} d s-1 \otimes 1 \\
&= \frac{1}{n} \int_{\mathbb{R}_{+}}\left(\nabla_{s} F\right) \sharp\left(\nabla_{s} F\right)^{*} d s-1 \otimes 1 \\
&= \frac{1}{n} \sum_{k, q=1}^{n} \sum_{p=0}^{(k \wedge q)-1} \sum_{r=0}^{n-(k \vee q)} \mathbb{1}_{\{n-1-p-r>0\}} \\
& \text { (13) } \quad=\frac{1}{n} \sum_{p, r=0}^{n-1} \sum_{k, q=0}^{n-1-p-r} \mathbb{1}_{\{n-1-p-r>0\}} \cdot I_{k+q} \otimes I_{2(n-1-p-r)-k-q}(f \stackrel{p+r+1}{\sim} f) .
\end{aligned}
$$

Grouping all occuring bi-integrals by the order of the contraction, one arrives at

$$
\begin{aligned}
\int_{\mathbb{R}_{+}} \nabla_{s}\left(N_{0}^{-1} F\right) \sharp\left(\nabla_{s} F\right)^{*} d s- & \otimes 1 \\
= & \frac{1}{n} \sum_{u=1}^{n-1} \sum_{v=0}^{2(n-u)} c_{u, v} I_{v} \otimes I_{2(n-u)-v}(f \stackrel{u}{\sim} f),
\end{aligned}
$$

where the $c_{u, v}$ are positive constants depending solely on $u$ and $v$. Taking the trace of the square of (14) and using the Wigner bisometry (5) yields

$$
\begin{aligned}
\varphi \otimes \varphi\left(\mid \int_{\mathbb{R}_{+}} \nabla_{s}\left(N_{0}^{-1} F\right)\right. & \left.\sharp\left(\nabla_{s} F\right)^{*} d s-\left.1 \otimes 1\right|^{2}\right) \\
& =\frac{1}{n^{2}} \sum_{u=1}^{n-1} \sum_{v=0}^{2(n-u)} c_{u, v}^{2}\|f \stackrel{u}{ } f\|_{L^{2}\left(\mathbb{R}_{+}^{2 n-2 u}\right)}^{2} \\
& \leq \frac{1}{n^{2}} \max _{1 \leq u \leq n-1}\left\{\sum_{v=0}^{2(n-u)} c_{u, v}^{2}\right\} \sum_{u=1}^{n-1}\|f \stackrel{u}{\sim} f\|_{L^{2}\left(\mathbb{R}_{+}^{2 n-2 u}\right)}^{2} .
\end{aligned}
$$

As is well known,

$$
\sum_{u=1}^{n-1}\|f \stackrel{u}{\sim} f\|_{L^{2}\left(\mathbb{R}_{+}^{2 n-2 u}\right)}^{2}=\varphi\left(F^{4}\right)-2,
$$

so that it only remains to evaluate the maximum. To this end, the constants $c_{u, v}$ will be computed explicitly. By carefully comparing (13) with (14), one sees that $c_{u, v}$ is given by the cardinality of the set of all quadruples $(p, r, k, q)$ satisfying the following conditions:

$$
\begin{array}{ll}
0 \leq p, r \leq n-1 ; & 0 \leq k, q \leq n-1-p-r ; \\
k+q=v ; & p+r=u-1 ; \\
p+r<n-1 . &
\end{array}
$$

By reindexing the second sum in (13) via the transformation $\left(k^{\prime}, q^{\prime}\right)=(n-1-p-$ $r-k, n-1-p-r-q)$, we see that $c_{u, v}=c_{u, 2(n-u)-v}$, thus only the constants $c_{u, v}$ for which $v \leq n-u$ need to be computed explicitly. Fix $u$ and $v$. Then, there are 
$u$ couples $(p, r)$ satisfying $p+r=u-1$, namely $(0, u-1),(1, u-2), \ldots,(u-1,0)$. Likewise, there are $v+1$ couples $(k, q)$ satisfying $k+q=v$. Therefore,

$$
c_{u, v}= \begin{cases}u(v+1) & \text { if } v \leq n-u \\ u(2(n-u)-v+1) & \text { if } v>n-u\end{cases}
$$

This yields

$$
\begin{aligned}
\sum_{v=0}^{2(n-u)} c_{u, v}^{2} & =\sum_{v=0}^{n-u} u^{2}(v+1)^{2}+\sum_{v=n-u+1}^{2(n-u)} u^{2}(2(n-u)-v+1)^{2} \\
& =\sum_{v=0}^{n-u} u^{2}(v+1)^{2}+\sum_{v=0}^{n-u-1} u^{2}(v+1)^{2} \\
& =\frac{1}{3} u^{2}(n-u)(n-u+1)(2(n-u)+1)+u^{2}(n-u+1)^{2} \\
& =P_{n}(u) .
\end{aligned}
$$

Straightforward analysis shows that the polynomial $P_{n}$ has exactly one maximum in the interval $(1, n-1)$ attained at $u_{0}$ as defined in (11). Therefore, to maximize $P_{n}$, one has to select the closest integer to $u_{0}$.

Combining Theorem 3.7 with the bound appearing in (7) and applying the CauchySchwarz inequality immediately yields the following quantitative free Fourth Moment Theorem.

Corollary 3.8. Let $n \geq 2$ be a natural number and $F=I_{n}(f)$, where $f$ is a symmetric function in $L^{2}\left(\mathbb{R}_{+}^{n}\right)$ such that $\|f\|_{L^{2}\left(\mathbb{R}_{+}^{n}\right)}^{2}=1$. Let $S$ be standard semicircular random variable. Then it holds that,

$$
d_{\mathcal{C}_{2}}(F, S) \leq \frac{\sqrt{C_{n}}}{2} \sqrt{\varphi\left(F^{4}\right)-2}
$$

where $C_{n}$ is the constant appearing in Theorem 3.7.

Remark 3.9.

1. It holds that $C_{2}=\frac{3}{2}$, so that Corollary 3.8 becomes

$$
d_{\mathcal{C}_{2}}\left(I_{2}(f), S\right) \leq \frac{1}{2} \sqrt{\frac{3}{2}} \sqrt{\varphi\left(I_{2}(f)^{4}\right)-2}
$$

which is precisely the conclusion of [KNPS12, Corollary 1.12]. The next few values of $C_{n}$ are given by $C_{3}=2, C_{4}=\frac{19}{4}$ yielding

$$
d_{\mathcal{C}_{2}}\left(I_{3}(f), S\right) \leq \frac{1}{\sqrt{2}} \sqrt{\varphi\left(I_{3}(f)^{4}\right)-2}
$$

and

$$
d_{\mathcal{C}_{2}}\left(I_{4}(f), S\right) \leq \frac{\sqrt{19}}{4} \sqrt{\varphi\left(I_{4}(f)^{4}\right)-2},
$$

where $f$ is of course always chosen appropriately to be symmetric and an element of $L^{2}\left(\mathbb{R}_{+}^{n}\right)$ for each $n=2,3,4$. 
2. In general, a straightforward analysis shows that $C_{n}$ grows with $n$. In the commutative case, when bounding the distance between a multiple Wiener integral of any order and the standard Gaussian distribution by means of the fourth moment, the constants appearing in the bounds do not depend on the order of the multiple integral (see for example [NP09b]). If such a dimension-free bound also holds in the free case is not known and this question is left for future research.

3. As stated in the introduction, convergence of the fourth moment to 2 also implies convergence of multiple integral with mirror-symmetric kernels towards the semicircular distribution. As one needs the function $f$ to be symmetric in Theorem 3.7, it is natural to ask if the bound (10) also holds for mirror-symmetric kernels. As the following counterexample shows, this is not true. Divide $[0,1]$ into $N$ intervals $I_{1}, I_{2}, \ldots, I_{N}$ of equal length $\frac{1}{N}$ and define the function

$$
f_{N}\left(x_{1}, x_{2}, x_{3}\right)=\sqrt{N} \sum_{k=1}^{N} \mathbb{1}_{I_{k} \times I_{k}}\left(x_{1}, x_{3}\right)
$$

on $[0,1]^{3}$. Observe that $f_{N}$ is a mirror-symmetric function in $L^{2}\left([0,1]^{3}\right)$. Then,

$$
I_{3}\left(f_{N}\right)=\sqrt{N} \sum_{k=1}^{N} I_{1}\left(\mathbb{1}_{I_{k}}\right) I_{1}(1) I_{1}\left(\mathbb{1}_{I_{k}}\right) .
$$

It is easy to check that $\varphi\left(I_{3}\left(f_{N}\right)^{2}\right)=1$ and

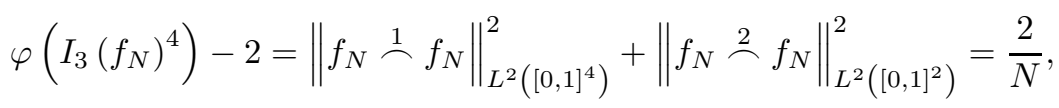

implying (by the free Fourth Moment Theorem of [KNPS12]) that the sequence $\left\{I_{3}\left(f_{N}\right): N \geq 1\right\}$ converges in distribution to the standard semicircular law. Furthermore,

$$
\begin{aligned}
\nabla_{t} I_{3}\left(f_{N}\right)=\sqrt{N} \sum_{k=1}^{N}\left[\mathbb{1}_{I_{k}}(t) \otimes\right. & I_{1}(1) I_{1}\left(\mathbb{1}_{I_{k}}\right) \\
& \left.+I_{1}\left(\mathbb{1}_{I_{k}}\right) \otimes I_{1}\left(\mathbb{1}_{I_{k}}\right)+I_{1}\left(\mathbb{1}_{I_{k}}\right) I_{1}(1) \otimes \mathbb{1}_{I_{k}}(t)\right] .
\end{aligned}
$$

As $f_{N}$ is a sum of products of non-negative indicator functions, the quantity

$$
\varphi \otimes \varphi\left(\left|\int_{\mathbb{R}_{+}} \nabla_{s}\left(N_{0}^{-1} I_{3}\left(f_{N}\right)\right) \sharp\left(\nabla_{s} I_{3}\left(f_{N}\right)\right)^{*} d s-1 \otimes 1\right|^{2}\right)
$$

is a sum of non-negative terms. Hence, if one of these terms can be proven not to converge to zero, the entire quantity must be bounded away from zero as well. One of the summands appearing is $\sqrt{N} \sum_{k=1}^{N} I_{1}\left(\mathbb{1}_{I_{k}}\right) \otimes I_{1}\left(\mathbb{1}_{I_{k}}\right)$. It holds that

$$
\begin{aligned}
\left(\sqrt{N} \sum_{k=1}^{N} I_{1}\left(\mathbb{1}_{I_{k}}\right) \otimes I_{1}\left(\mathbb{1}_{I_{k}}\right)\right) \sharp & \left(\sqrt{N} \sum_{k=1}^{N} I_{1}\left(\mathbb{1}_{I_{k}}\right) \otimes I_{1}\left(\mathbb{1}_{I_{k}}\right)\right)^{*} \\
& =N \sum_{k, q=1}^{N} I_{1}\left(\mathbb{1}_{I_{k}}\right) I_{1}\left(\mathbb{1}_{I_{q}}\right) \otimes I_{1}\left(\mathbb{1}_{I_{q}}\right) I_{1}\left(\mathbb{1}_{I_{k}}\right)
\end{aligned}
$$


and a straightforward calculation shows that

$$
\varphi \otimes \varphi\left(\left|N \sum_{k, q=1}^{N} I_{1}\left(\mathbb{1}_{I_{k}}\right) I_{1}\left(\mathbb{1}_{I_{q}}\right) \otimes I_{1}\left(\mathbb{1}_{I_{q}}\right) I_{1}\left(\mathbb{1}_{I_{k}}\right)\right|^{2}\right)=1+\frac{3}{N},
$$

which does not go to zero as $N$ goes to infinity. In total, it holds that $\varphi\left(I_{3}\left(f_{N}\right)^{4}\right)$ $2 \rightarrow 0$ as $N$ goes to infinity, but the quantity (15) is strictly greater than 1 for all $N$, hence proving that the quantity (15) can not be controlled by the fourth moment. Therefore, Theorem 3.7 can not be extended to mirror-symmetric kernels.

In the commutative case, the classical Nualart-Ortiz-Latorre equivalence criterion for normal convergence of multiple Wiener integrals $F_{k}=I_{n}^{W}\left(f_{k}\right)$ reads

$$
\int_{\mathbb{R}_{+}}\left(D_{s} F_{k}\right)^{2} d s \rightarrow n \text { in } L^{2}(\Omega), \text { as } k \rightarrow \infty
$$

where $D$ denotes the Malliavin gradient and $\Omega$ stands for the underlying probability space (see [NOL08]). For Wigner integrals, an analogue of this criterion was only known to hold in the second chaos (see [KNPS12, Theorem 4.8]). Theorem 3.7 extends this to any order of chaos. Therefore, all equivalent criteria for normal convergence of Wiener integrals have now free analogues for convergence of Wigner integrals towards a semicircular distribution. For the sake of completeness, we collect these analogues in the following Theorem.

Theorem 3.10. Let $n \geq 2$ be a natural number and let $\left\{f_{k}: k \geq 1\right\}$ be a sequences of symmetric functions in $L^{2}\left(\mathbb{R}_{+}^{n}\right)$ such that, for all $k \geq 1,\left\|f_{k}\right\|_{L^{2}\left(\mathbb{R}_{+}^{n}\right)}=1$. For any $k \geq 1$, denote $F_{k}=I_{n}\left(f_{k}\right)$. Then, the following conditions are equivalent:

(i) The sequence $\left\{F_{k}: k \geq 1\right\}$ converges in law to the standard semicircular distribution.

(ii) As $k$ tends to infinity, $\varphi\left(F_{k}^{4}\right) \rightarrow 2$.

(iii) For all $1 \leq p \leq n-1$, as $k$ tends to infinity, $\left\|f_{k} \stackrel{p}{f_{k}}\right\|_{L^{2}\left(\mathbb{R}_{+}^{2 n-2 p}\right)} \rightarrow 0$.

(iv) As $k$ tends to infinity,

$$
\int_{\mathbb{R}_{+}}\left(\nabla_{s} F_{k}\right) \sharp\left(\nabla_{s} F_{k}\right)^{*} d s \rightarrow n \cdot 1 \otimes 1 \quad \text { in } \quad L^{2}(\mathcal{S} \otimes \mathcal{S}, \varphi \otimes \varphi) .
$$

Proof. The equivalences $(i) \Leftrightarrow(i i) \Leftrightarrow(i i i)$ and the implication $(i v) \Rightarrow(i)$ follow from [KNPS12, Theorem 1.6, Theorem 1.10]. The missing implication $(i i) \Rightarrow(i v)$ follows from the main result of this section, namely Theorem 3.7.

\section{Quantifying the Free Breuer-Major theorem}

Our main results can be used to provide Berry-Esseen bounds for a free version of the Breuer-Major theorem (see [KNPS12]) for the free fractional Brownian motion. This can be regarded as a free analog of [NP09b, Theorem 4.1]. The free fractional 
Brownian motion $S^{H}$ with index $H \in(-1,1)$ is defined as a centered semicircular process with covariance function

$$
\varphi\left(S_{t}^{H} S_{s}^{H}\right)=\frac{1}{2}\left(t^{2 H}+s^{2 H}-|t-s|^{2 H}\right) .
$$

As is well-known (see for example [BS98] or [NS06]), the orthogonal polynomials associated to the semicircular distribution are the Chebyshev polynomials $U_{n}$ of the second kind defined on $[-2,2]$ by the recurrence relations $U_{0}(x)=1, U_{1}(x)=x$, and for $n \geq 2$,

$$
U_{n+1}(x)=x U_{n}(x)-U_{n-1}(x) .
$$

For $n \in \mathbb{N}$, define the increment sequence $\left\{X_{k}=S_{k+1}^{H}-S_{k}^{H}: k \geq 0\right\}$. Straightforward calculations show that the autocovariance function $\rho_{H}(k)$ is given by

$$
\rho_{H}(k)=\varphi\left(X_{0} X_{k}\right)=\frac{1}{2}\left(|k+1|^{2 H}+|k-1|^{2 H}-2|k|^{2 H}\right) .
$$

Furthermore, define $\left\{V_{m}: m \geq 1\right\}$ as

$$
V_{m}=\frac{1}{\sqrt{m}} \sum_{k=0}^{m-1} U_{n}\left(X_{k}\right) .
$$

With these definitions in place, we can now state the announced Berry-Esseen bounds.

Theorem 4.1. With the above notation prevailing, suppose that there exists an integer $n \geq 1$ such that $\sigma^{2}=\sum_{k \in \mathbb{Z}}\left|\rho_{H}(k)\right|^{n}<\infty$. Then, there exists a positive constant $C_{n, H}$ such that

$$
d_{\mathcal{C}_{2}}\left(\frac{V_{m}}{\sigma}, \mathcal{S}(0,1)\right) \leq C_{n, H} m^{\alpha(n, H)},
$$

where the function $\alpha(n, H)$ is given by

$$
\alpha(n, H)= \begin{cases}m^{-\frac{1}{2}} & \text { if } H \in\left(0, \frac{1}{2}\right], \\ m^{H-1} & \text { if } H \in\left[\frac{1}{2}, \frac{2 n-3}{2 n-2}\right], \\ m^{n H-n+\frac{1}{2}} & \text { if } H \in\left[\frac{2 n-3}{2 n-2}, \frac{2 n-1}{2 n}\right) .\end{cases}
$$

Proof. It is well known (see e.g. [Nou12, Proposition 2.5]), that the (non-free) fractional Brownian motion can be represented as a Wiener integral with respect to a standard Brownian motion as

$$
B_{t}^{H}=\int_{0}^{t} K_{H}(t, u) d W_{u}
$$

where the kernel $K_{H}(\cdot, \cdot)$ is explicit (see e.g. [Nou12, Proposition 2.5]). Using the correspondence between Wiener and Wigner integrals, it also holds that

$$
S_{t}^{H}=\int_{0}^{t} K_{H}(t, u) d S_{u}
$$

Indeed, this can be verified by checking that the covariance function of the above integral coincides with the one of the free fractional Brownian motion. Then, denoting

$$
f_{k, m, H}=m^{H}\left(K_{H}\left(\frac{k+1}{m}, \cdot\right) \mathbb{1}_{\left[0, \frac{k+1}{m}\right]}-K_{H}\left(\frac{k}{m}, \cdot\right) \mathbb{1}_{\left[0, \frac{k}{m}\right]}\right)
$$


it holds that

$$
V_{m}=\frac{1}{\sqrt{m}} \sum_{k=0}^{m-1} U_{n}\left(I_{1}\left(f_{k, m, H}\right)\right)
$$

Observe that $\left\|f_{k, m, H}\right\|_{L^{2}\left(\mathbb{R}_{+}\right)}=1$ so that

$$
V_{m}=\frac{1}{\sqrt{m}} \sum_{k=0}^{m-1} I_{n}\left(f_{k, m, H}^{\otimes n}\right)=I_{n}\left(\frac{1}{\sqrt{m}} \sum_{k=0}^{m-1} f_{k, m, H}^{\otimes n}\right) .
$$

Define

$$
g_{n, m, H}=\frac{1}{\sigma \sqrt{m}} \sum_{k=0}^{m-1} f_{k, m, H}^{\otimes n} .
$$

Applying Corollary 3.8 to $V_{m}$, we get

$$
\begin{aligned}
& d_{\mathcal{C}_{2}}\left(\frac{V_{m}}{\sigma}, \mathcal{S}(0,1)\right) \leq \frac{\sqrt{C_{n}}}{2} \sqrt{\varphi\left(I_{n}\left(g_{n, m, H}\right)^{4}\right)-2} \\
& =\frac{\sqrt{C_{n}}}{2} \sqrt{\sum_{u=1}^{n-1}\left\|g_{n, m, H} \stackrel{u}{\sim} g_{n, m, H}\right\|_{L^{2}\left(\mathbb{R}_{+}^{2 n-2 u}\right)}^{2}} .
\end{aligned}
$$

From here, one can evaluate and estimate the contraction norms similarly as in the proof of [NP09b, Theorem 4.1].

Acknowledgments. The authors wish to thank Roland Speicher for providing the counterexample appearing in Remark 3.9 and Tobias Mai and Roland Speicher for several stimulating discussions. S. Campese was partially supported by ERC grant 277742 Pascal.

\section{REFERENCES}

[ACP14] Ehsan Azmoodeh, Simon Campese, and Guillaume Poly. Fourth Moment Theorems for Markov diffusion generators. Journal of Functional Analysis, 266(4):2341-2359, 2014.

[Bou15] Solesne Bourguin. Poisson convergence on the free Poisson algebra. Bernoulli. Official Journal of the Bernoulli Society for Mathematical Statistics and Probability, 21(4):2139-2156, 2015.

[Bou16] Solesne Bourguin. Vector-valued semicircular limits on the free Poisson chaos. Electronic Communications in Probability, 21(55):1-11, 2016.

[BP14a] Solesne Bourguin and Giovanni Peccati. Portmanteau inequalities on the Poisson space: mixed regimes and multidimensional clustering. Electronic Journal of Probability, 19:no. 66, 42, 2014.

[BP14b] Solesne Bourguin and Giovanni Peccati. Semicircular limits on the free Poisson chaos: Counterexamples to a transfer principle. Journal of Functional Analysis, 267(4):963997, August 2014.

[BS98] Philippe Biane and Roland Speicher. Stochastic calculus with respect to free Brownian motion and analysis on Wigner space. Probability Theory and Related Fields, 112(3):373-409, 1998.

[CNPP16] Simon Campese, Ivan Nourdin, Giovanni Peccati, and Guillaume Poly. Multivariate Gaussian approximations on Markov chaoses. Electronic Communications in Probability, 21, 2016.

[DN12] Aurélien Deya and Ivan Nourdin. Convergence of Wigner integrals to the tetilla law. ALEA. Latin American Journal of Probability and Mathematical Statistics, 9:101-127, 2012.

[DNN13] Aurélien Deya, Salim Noreddine, and Ivan Nourdin. Fourth moment theorem and $q$ Brownian chaos. Communications in Mathematical Physics, 321(1):113-134, 2013. 
[HP00] Fumio Hiai and Dénes Petz. The semicircle law, free random variables and entropy, volume 77 of Mathematical Surveys and Monographs. American Mathematical Society, Providence, RI, 2000.

[KNPS12] Todd Kemp, Ivan Nourdin, Giovanni Peccati, and Roland Speicher. Wigner chaos and the fourth moment. The Annals of Probability, 40(4):1577-1635, 2012.

[Led12] M. Ledoux. Chaos of a Markov operator and the fourth moment condition. The Annals of Probability, 40(6):2439-2459, November 2012. Zentralblatt MATH identifier: 06114704 .

[NOL08] D. Nualart and S. Ortiz-Latorre. Central limit theorems for multiple stochastic integrals and Malliavin calculus. Stochastic Processes and their Applications, 118(4):614-628, 2008.

[Nou12] Ivan Nourdin. Selected aspects of fractional Brownian motion, volume 4 of Bocconi $\&$ Springer Series. Springer, Milan, 2012.

[NP05] David Nualart and Giovanni Peccati. Central limit theorems for sequences of multiple stochastic integrals. The Annals of Probability, 33(1):177-193, 2005.

[NP09a] Ivan Nourdin and Giovanni Peccati. Noncentral convergence of multiple integrals. The Annals of Probability, 37(4):1412-1426, July 2009.

[NP09b] Ivan Nourdin and Giovanni Peccati. Stein's method on Wiener chaos. Probability Theory and Related Fields, 145(1-2):75-118, 2009.

[NP13] Ivan Nourdin and Giovanni Peccati. Poisson approximations on the free Wigner chaos. The Annals of Probability, 41(4):2709-2723, 2013.

[NPR10] Ivan Nourdin, Giovanni Peccati, and Anthony Réveillac. Multivariate normal approximation using Stein's method and Malliavin calculus. Annales de l'Institut Henri Poincaré Probabilités et Statistiques, 46(1):45-58, 2010.

[NPS13] Ivan Nourdin, Giovanni Peccati, and Roland Speicher. Multi-dimensional Semicircular Limits on the Free Wigner Chaos. In Robert C. Dalang, Marco Dozzi, and Francesco Russo, editors, Seminar on Stochastic Analysis, Random Fields and Applications VII, number 67 in Progress in Probability, pages 211-221. Springer Basel, January 2013.

[NS06] Alexandru Nica and Roland Speicher. Lectures on the combinatorics of free probability, volume 335 of London Mathematical Society Lecture Note Series. Cambridge University Press, Cambridge, 2006.

[NT14] Ivan Nourdin and Murad S. Taqqu. Central and non-central limit theorems in a free probability setting. Journal of Theoretical Probability, 27(1):220-248, 2014.

[Pec11] Giovanni Peccati. The Chen-Stein method for Poisson functionals. arXiv:1112.5051, December 2011.

[PSTU10] G. Peccati, J. L. Solé, M. S. Taqqu, and F. Utzet. Stein's method and normal approximation of Poisson functionals. The Annals of Probability, 38(2):443-478, 2010.

[PT05] Giovanni Peccati and Ciprian A. Tudor. Gaussian limits for vector-valued multiple stochastic integrals. In Séminaire de Probabilités XXXVIII, volume 1857 of Lecture Notes in Math., pages 247-262. Springer, Berlin, 2005.

[PT13] Giovanni Peccati and Christoph Thäle. Gamma limits and $u$-statistics on the Poisson space. ALEA. Latin American Journal of Probability and Mathematical Statistics, 10(1):525-560, 2013.

[PZ10] Giovanni Peccati and Cengbo Zheng. Multi-dimensional Gaussian fluctuations on the Poisson space. Electronic Journal of Probability, 15:no. 48, 1487-1527, 2010.

[Tao12] Terence Tao. Topics in random matrix theory, volume 132 of Graduate Studies in Mathematics. American Mathematical Society, Providence, RI, 2012.

[VDN92] D. V. Voiculescu, K. J. Dykema, and A. Nica. Free random variables, volume 1 of CRM Monograph Series. American Mathematical Society, Providence, RI, 1992. A noncommutative probability approach to free products with applications to random matrices, operator algebras and harmonic analysis on free groups. 
Boston University, Department of Mathematics and Statistics, 111 Cummington Mall, Boston, MA 02215, USA

E-mail address: solesne.bourguin@gmail.com

University of Luxembourg, Mathematics Research Unit, 6, Rue Richard CoudenhoveKALERGI, 1359 LuXembourg, LuXembourG

E-mail address: simon.campese@uni.lu 